\title{
Septic arthritis due to underlying Scedosporium apiospermum chronic osteomyelitis. Report of one case and brief review of the literature
}

\author{
Jorge R. Mosqueira', Leslie M. Soto ${ }^{2}$, Jorge Soria ${ }^{1}$, Ana B. Bustamante ${ }^{2,3}$, \\ Jaime Cáceres ${ }^{4}$, Gloria M. Fuentes Rivera ${ }^{4}$ \\ ${ }^{1}$ Médico Cirujano. Universidad Peruana Cayetano Heredia. Lima, Perú. \\ ${ }^{2}$ Médico Infectólogo. Hospital Cayetano Heredia. Departamento de Medicina del Hospital Cayetano Heredia. Lima, Perú. \\ ${ }^{3}$ Medico Asistente del Departamento de Enfermedades Infecciosas, Tropicales y Dermatológicas del Hospital Cayetano Heredia. \\ Lima, Perú. \\ ${ }^{4}$ Servicio de Anatomía Patológica. Hospital Cayetano Heredia. Lima, Perú.
}

\begin{abstract}
Scedosporium spp. are saprophytic filamentous fungi isolated from soil, sewage and polluted water. Traumatic inoculation is a common way to become infected. We present a case of a patient with septic arthritis and chronic osteomyelitis due to Scedosporium apiospermum successfully treated with voriconazole. J Microbiol Infect Dis 2016;6(4): 87-90
\end{abstract}

Key words: Scedosporiosis, Scedosporium apiospermum, fungal arthritis, fungal osteomyelitis, voriconazole

\section{INTRODUCTION}

Scedosporium species have been implicated in a wide variety of life-threatening infections in humans, especially in immunocompromised hosts. Post-transplant patients are commonly involved; however, these fungi have been identified as an increasing cause of infection in immunocompetent individuals [1-4]. Inhalation of airborne fungal spores, traumatic inoculation and near-drowning incidents are common to become infected by Scedosporium spp. [1-3]. Clinical presentation depends on the interaction of host and pathogen. Infections caused by this agent have been associated with the following four clinical conditions: 1) mycetoma, 2) saprobic involvement/colonization of airways, and deep infections, which may be classified as 3 ) localized or 4) disseminated [2]. With this manuscript we suggest to consider the possibility of fungal infections, as Scedosporium apiospermum (teleomorph state Pseudallescheria boydii) [5], after any traumatic accident in endemic areas.

\section{CASE}

A 35 year-old immunocompetent latin american man, whose past medical history is significant for a right tibial plateau and fibula open fracture, oc- curred eight years ago in the amazon rainforest of Peru (Iquitos) after a motorcycle accident, was transferred to our private clinic after broad-spectrum antibiotic therapy and several diagnostic/therapeutic arthroscopic procedures, performed due to septic arthritis of his right knee.

The present illness is remarkable for a 15-day history of progressive right knee swelling, pain and difficulty walking despite taking ibuprofen. With these symptoms, which started during a business trip in Florida, he presented to the emergency room (ER) for first time at a Hospital in the United States of America, where an arthrocentesis of his right knee was performed. This procedure showed a turbid yellow fluid containing 68750 white blood cells (WBC) with $93 \%$ neutrophil count, $7.1 \mathrm{~g} / \mathrm{l}$ of protein level, $41 \mathrm{mg} / \mathrm{dl}$ of glucose level, negative gram staining, negative aerobic/anaerobic bacterial culture, negative acid fast bacilli (AFB) smear and negative for fungal elements. Surgical procedures as right knee arthroscopies, synovectomy, right knee irrigation and debridement were done showing signs consistent with septic arthritis. Indeed, findings of the magnetic resonance imaging (MRI) of the right tibia and fibula were suggestive of septic arthritis of the suprapatellar bursa, as well as underlying chronic osteomyelitis of the proximal tibia. Ini-

Correspondence: Dr. Jorge R. Mosqueira, Hospital Cayetano Heredia, Avenida Honorio Delgado No: 262, Urbanización Ingeniería, San Martín de Porres, Lima 31 ,Perú Email: jorge.mosqueira.s@alumni-upch.edu.pe Received: 09 May 2016; Accepted: 09 August 2016 
tial antibiotic therapy consisted of IV cefazolin were switched to vancomycin due to an allergic reaction. Levofloxacin was also used. After 5 days of hospitalization and moderate symptoms improvement he was discharged with a close follow-up and specific treatment previously mentioned. Later on, a new arthroscopy reported favorable results, although the symptoms had still persisted. Almost one month later the symptoms started, he went back to Peru.

Because of the persistence of symptoms after his arrival, he was admitted to a public hospital where was started on vancomycin and meropenem. Another three arthroscopies were performed with no significant findings and joint fluid was studied without isolating the causative organism. Symptoms worsened despite treatment, reason why the patient was transferred to our private clinic.

We repeated the diagnostic tests previously done, without positive findings regarding to the etiology. Nonetheless, a biopsy of the proximal tibia was performed, sending a sample for bacterial, fungal and Mycobacterium tuberculosis culture. In the meantime, he was started on IV vancomycin and IV meropenem. No antifungal therapy had been included until we got the diagnosis.

The continuous deformation of the right knee without other symptoms, unsuccessful antibiotic therapy, unknown etiology, negative aerobic/anaerobic bacterial culture, negative AFB testing, but a bone biopsy positive for granulomas in a country as Peru where there is a high prevalence of Tuberculosis (TB), led us to continue the current antibiotic therapy and initiate the standard empiric treatment for TB we use in Peru (isoniazid, rifampin, pyrazinamide, and ethambutol) while waiting for specific culture results.

After 12 days of continuous right knee irrigation, he developed a sinus tract opening at right knee joint with discharge of purulent material. He was unable to walk due to the progressive deformation of his right knee. However, since the septic arthritis was controlled, he was discharged 20 days after his admission with close follow-up. Vancomycin and meropenem were discontinued, holding the standard treatment for TB. Besides, he was started on PO levofloxacin and PO linezolid.

During his follow-up, despite optimal medical therapy the symptoms worsened. His right knee sinus tract had not been healed and around two months later a negative culture for Mycobacterium tuberculosis was reported. Therefore, due to his lack of sustained clinical improvement, the patient was readmitted to our clinic. A new arthroscopy was performed; in addition, bone tissue samples of the right tibia taken at this time were sent to our laboratory for analysis.

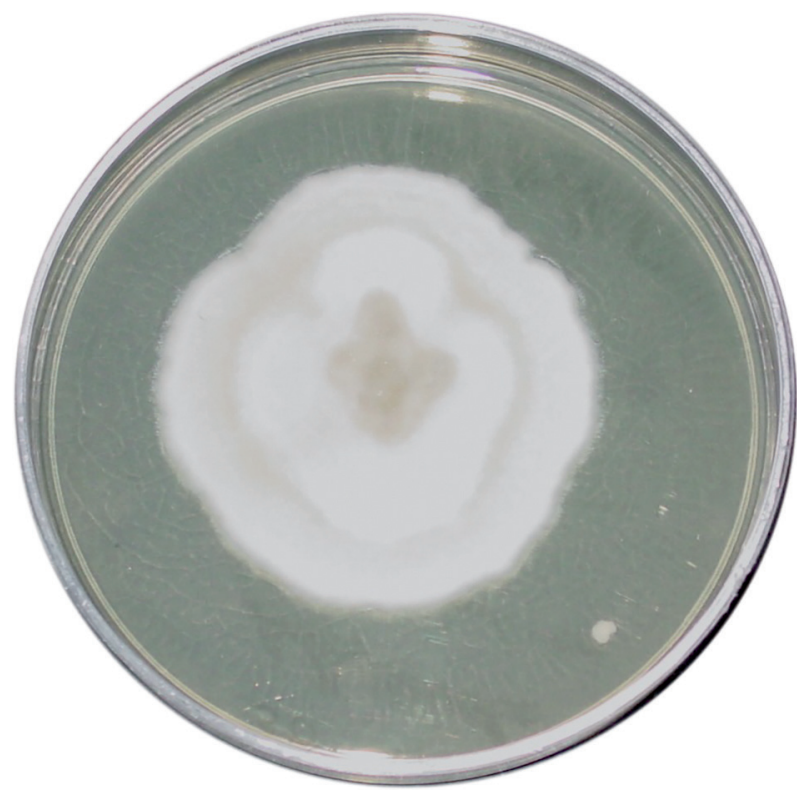

Figure 1. Scedosporium apiospermum culture morphology after 8 days of incubation on Sabouraud's dextrose agar at $25^{\circ} \mathrm{C}$.

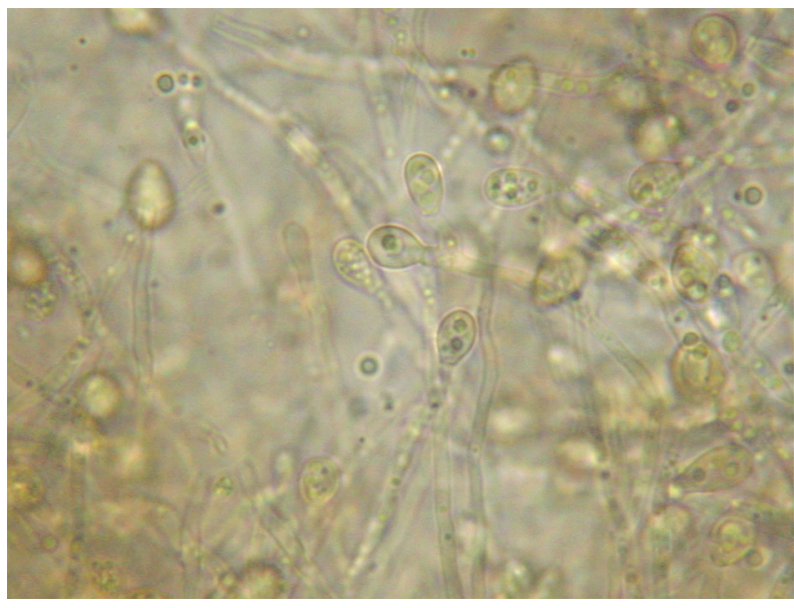

Figure 2. Microscope morphology showing single - celled, ovoid conidia with a truncate base on the top of the conidiogenous hyphae. Magnification, X1000.

Approximately four months later the symptoms started, we were informed that a spore of Scedosporium spp. was identified on bone sample and later by fungal culture (Figures 1 and 2). Hence, drug treatment for tuberculosis, levofloxacin and linezolid were discontinued. This patient was started on voriconazole $200 \mathrm{mg}$ bid showing good treat- 
ment response. An MRI of his right knee, done three months after antifungal therapy was established, demonstrated a significant improvement over previous images and favorable outcomes regarding chronic osteomyelitis of the proximal tibia. Additionally, femoral and patellar chronic osteomyelitis have been resolved.

\section{DISCUSSION}

Scedosporium spp. are saprophytic molds increasingly encountered as fungal pathogens that affect both immunocompromised and immunocompetent hosts $[1,3]$. These fungi are commonly found in soils containing high nitrogen content (agricultural or garden soils), sewage, polluted water, decaying matter (vegetation) and even in potted plants at home $[1,2,4,6]$, showing a worldwide distribution. In Peru, there is no epidemiological studies related to these fungi; however, in 2009 Somocurcio JG et al. reported the first two cases of pulmonary mycetoma by Scedosporium spp. identified in two patients with post-TB cavitary sequelae [7].

Our patient presented with septic arthritis due to an underlying chronic osteomyelitis. $\mathrm{He}$ is not a transplant recipient and no other risk factors for immunosuppression were identified (hematological malignancies, primary immunodeficiency disorders, human immunodeficiency virus (HIV) infection, and intravenous drug use). His past medical history of right knee trauma exposed to soil and decaying vegetation in the rain forest of Peru is epidemiologically important for this case. In addition, it is relevant that transcutaneous trauma is one way of transmission, including immunocompetent hosts [2].

Scedosporium spp. were diagnosed based on histopathology and culture. Histopathological study reveals acute and chronic inflammatory infiltrate affecting bone and soft tissue, findings consistent with acute and chronic osteomyelitis the presence of septate and ramified hyphae with abundant oval conidia are observed in one section, illustrated with PAS (periodic acid-Schiff) and GMS (Grocott methenamine silver) staining (Figures 3 and 4).

However, despite Scedosporium apiospermum complex shows more irregularly branching pattern and terminal or intercalary, globose chlamydospores, sometimes could not be distinguished morphologically from Fusarium spp. and Aspergillus spp. since all these fungi produce branched and septate hyphae [4]. Therefore, DNA sequencing (GenBank and the International Society for Human and Animal Mycology (ISHAM) reference database)
[3] was required to finally establish Scedosporium apiospermum as the causative organism.

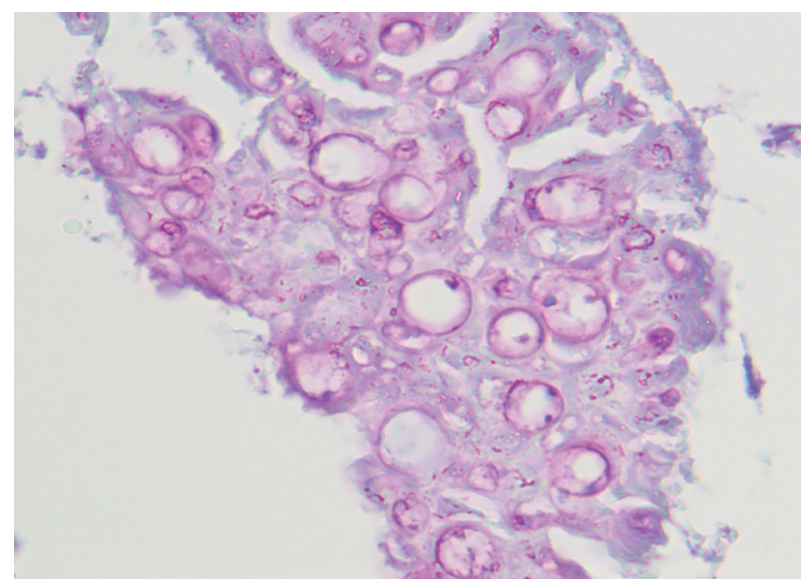

Figure 3. Mycrophotograph: numerous oval shape conidia and septate hyphae. Periodic acid-Schiff (PAS) staining.

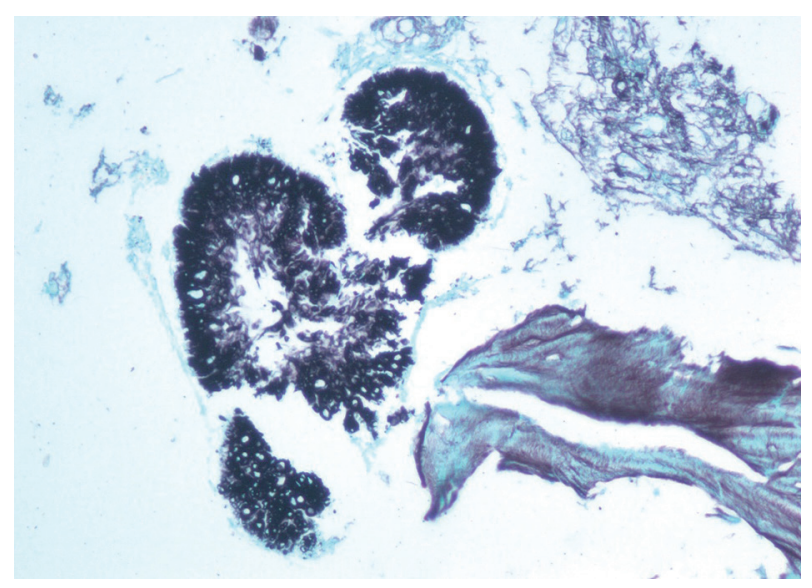

Figure 4. Mycrophotograh: numerous oval shape conidia and septate hyphae. Grocott methenamine silver (GMS) staining.

Scedosporium apiospermum is part of the Scedosporium apiospermum complex which also involves $S$. aurantiacum and $S$. boydii, these three fungi are the most frequent species isolated among clinical samples [3]. Scedosporium apiospermum complex and Lamentospora prolificans accounts for most infections in humans [1], producing disseminated and life-threatening infections specially in immunocompromised patients (transplant recipients, human immunodeficiency virus (HIV) infection, hematological malignancies, primary immunodeficiency disorders and intravenous drug use) [1-3,8] Commonly, infection can be acquired by inhalation of fungal spores, traumatic inoculation and neardrowning incidents [1-3]. 
In general, Scedosporium spp. can invade any tissue and cause a broad spectrum of diseases, including septic arthritis, osteomyelitis, soft tissue infection, ophthalmic infections, sinusitis, pneumonia, meningitis and brain abscesses (central nervous system), endocarditis, and disseminated infection $[2,8]$. Gelabert-González M et al. reported a case of Scedosporium apiospermum brain abscess that carries a high mortality rate and reviewed forty previously reported cases since 1977 to 2009 [9].

Localized cutaneous and subcutaneous infections are the most common clinical conditions in immunocompetent hosts $[8,10]$. Usually the lower extremities are affected after traumatic penetration with contaminated decaying matter. Maduromycosis is a localized and chronic local suppurative fungal infection of the soft tissue ("Madura foot", pedal mycetoma near Madura, India) produced by $S$. apiospermum $[8,10]$.

Osteoarticular involvement (arthritis or osteomyelitis) after a penetrating trauma is rare. MKT To, et al. reported an immunocompetent woman with S. apiospermum septic arthritis of the right hand, treated with radical debridement, arthrodesis of the wrist and postoperative itraconazole as longterm therapy [11]. In 2001, Tirado-Miranda R et al. reported an immunocompetent man with septic arthritis of his right knee due to $S$. apiospermum; in addition, reviewed and compared twelve previously reported cases finding six patients with good prognosis, three patients with limited range of motion of the affected joint after treatment, two patients were submitted to arthrodesis and one patient developed a disseminated condition [12].

In our patient, the diagnosis of $S$. apiospermum chronic osteomyelitis and septic arthritis of the right knee was established. Therefore, he was started on Voriconazole $200 \mathrm{mg}$ bid, since this drug is the standard treatment in the management of Scedosporium spp. showing clinical efficacy and good tolerance $[10,13]$. Five months later, the patient showed an excellent clinical response, his right knee was taking shape again and was able to walk alone using a walking stick. Eight months later, he still has some limited range of motion of his right knee; however, we keep following up with him once a month just make sure the treatment is effective. In the follow-up, we measure the voriconazole blood levels because is an effective method to improve efficacy and safety in this clinical setting $[10,13]$.

The main limitation of this case was to reach the diagnosis. The fact that the patient is immuno- competent limited the search for opportunistic infections; however, the endemic area and the low frequency of similar cases allowed us to expand the diagnostic possibilities.

\section{CONCLUSION}

In Peru, this is the third case of Scedosporium spp. reported and the first case of Scedosporium apiospermum as the causative organism of osteomyelitis and septic arthritis in an immunocompetent patient following a penetrating trauma. Certainly, osteoarticular disease is very rare; nonetheless, Scedosporium spp. can affect any tissue and accounts for an important pathogen with worldwide distribution. Immunocompromised patients, especially post-transplant recipients, are at higher risk to develop the infection; although, immunocompetent patients can be infected as well.

It is very important to consider the possibility of fungal infections after any traumatic accident in endemic areas; as well as to become acquainted with the epidemiology of these pathogens. We did not find any paper that mentions the relationship between the formation of granulomas and Scedoporium spp. infections. However, because of granulomas can be identified on histopathological examination of fungal-infected tissues, if we observe granulomas, as were reported on bone tissue in our case, it could be relevant to think about and rule out fungi. Therefore, we suggest taking into account these findings in clinical practice and future reports.

\section{Acknowledgement}

We acknowledge Edgar Neyra, MSc and biologist from the Department of Mycology at Instituto de Medicina Tropical Alexander Von Humboldt, for identifying molecularly the fungus reported on this paper.

Declaration of Conflicting Interests: The authors declare that they have no conflict of interest.

Financial Disclosure: No financial support was received.

\section{REFERENCES}

1. Subedi S, Chen SC.-A. Epidemiology of Scedosporiosis. Curr Fungal Infect Rep 2015; 9:275-284.

2. Cortez KJ, Roilides E, Quiroz-Telles F, et al. Infections caused by Scedosporium Scedosporium spp. Clin Microbiol Rev 2008; 21:157-97. 
3. Meyer W, Guarro J. Current Status in Diagnosis of Scedosporium Infections: What is the impact of New Molecular Methods? Curr Fungal Infect Rep 2014; 8:220-226.

4. Giraud S, Bouchara J-P. Scedosporium apiospermun Complex: Diagnosis and Species Identification. Curr Fungal Infect Rep 2014; 8(3):211-219.

5. Gilgado F, Gene J, Cano J, et al. Heterothallism in Scedosporium apiospermum and description of its teleomorph Pseudallescheria apiosperma sp. nov. Med Mycol 2010; 48:122-8.

6. Rofe NE, Haddad TJ, Wills TS. Management of Scedosporium apiospermum in a pre- and post-lung transplant patient with cystic fibrosis. Medical Mycology Case Reports 2013; 2:3739.

7. Somocurcio JG, Sotomayor A, et al. Micetoma pulmonar por Scedosporium spp., Reporte de dos casos. Rev Peru Med Exp Salud Publica 2009; 26(3):395-99.

8. Angelini A, Drago G, Ruggieri P. Post-tsunami primary Scedosporium apiospermum osteomyelitis of the knee in an immunocompetent patient. Int J Infect Dis 2013; 17.
9. Gelabert-González M, Lovo-Taboada J, et al. Absceso cerebral por Scedosporium apiospermum. Presentación de un caso con revisión de la literatura. Neurocirugía 2010; 21(2):125-131.

10. Henao-Martínez AF, Castillo-Mancilla JR, Barron MA, Nichol AC. Combination Antifungal Therapy in the Treatment of Scedosporium apiospermum Central Nervous System Infections. Case Rep Infect Dis 2013; 2013:589490.

11. MKT To, PL Ho, WY Ip. Scedosporium apiospermun Causing Septic Arthritis of the Hand in an Immunocompetent Patient. Hong Kong J Orthop Surg 2005; 9:73-78.

12. Tirado-Miranda R, Solera-Santos J, et al. Septic Arthritis Due to Scedosporium apiospermum: Case Report and Review. J Infect 2001; 43:210-212.

13. Muñóz P, Marín M, Tornero P, et al. Successful Outcome of Scedosporium apiospermum Disseminated Infection Treated with Voriconazole in a Patient Receiving Corticosteroid Therapy. Clin Infect Dis 2000; 31: 1499-1501. 